Zhang, T., Wu, Q. and Zhang, Z. (2020). Probable pangolin origin of SARS-CoV-2 associated with the COVID-19 outbreak. Curr Biol 30, 1346-1351.e2. doi:10.1016/j.cub.2020.03.022

Zhong, X., Harris, G., Smirnova, L. et al. (2020). Antidepressant paroxetine exerts developmental neurotoxicity in an iPSC derived 3D human brain model. Front Cell Neurosci 14, 25. doi:10.3389/fncel.2020.00025

Zhou, H., Chen, X., Hu, T. et al. (2020). A novel bat coronavirus closely related to SARS-CoV-2 contains natural insertions at the S1/S2 cleavage site of the spike protein. Curr Biol 30, 2196-2203.e3. doi:10.1016/j.cub.2020.05.023

Zhou, L., Zhang, M., Wang, J. et al. (2020). Sars-Cov-2: Underestimated damage to nervous system. Travel Med Infect Dis 36, 101642. doi:10.1016/j.tmaid.2020.101642

\section{Conflict of interest}

$\mathrm{TH}$ and $\mathrm{HH}$ are named inventors on a patent by Johns Hopkins University on the production of mini-brains (also called BrainSpheres), which is licensed to AxoSim, New Orleans, LA, USA. $\mathrm{TH}, \mathrm{LS}$ and HH consult AxoSim, and TH is a shareholder.

\section{Acknowledgment}

This work was financially supported by NIH R21 135527 , R01AI52688 and through the generosity of the collective community of donors to the Johns Hopkins University School of Medicine for COVID-19 research. Expert editing of the article by Mike Hughes is gratefully appreciated.

\title{
Non-aversive Mouse Handling Techniques: A Survey on the Acceptance and Concerns of the Animal Facility and Scientific Staff
}

\author{
Katharina Hohlbaum ${ }^{1}$, Melanie Humpenöder ${ }^{1}$, Stefan Nagel-Riedasch ${ }^{2}$, Kristina Ullmann ${ }^{2,3}$ \\ and Christa Thöne-Reineke1 \\ ${ }^{1}$ Institute of Animal Welfare, Animal Behavior, and Laboratory Animal Science, Department of Veterinary Medicine, Freie Universität Berlin, Berlin, \\ Germany; ${ }^{2}$ Research Facilities for Experimental Medicine (FEM), Charité - Universitätsmedizin Berlin, Berlin, Germany; ${ }^{3}$ Charité 3R, Charité - \\ Universitätsmedizin Berlin, Berlin, Germany
}

Routine laboratory procedures such as handling can cause stress and anxiety in animals, which in turn compromise animal welfare and are confounding factors in animal experimentation (Balcombe et al., 2004; Bailey, 2017). This underlines the need to develop and implement appropriate refinement strategies for animal handling techniques to minimize stress of animals when interacting with humans.

Laboratory mice are usually picked up by their tails. However, a decade ago, this traditional handling technique was refined by Hurst and West (2010) by introducing tunnel and cup handling. If a mouse is picked up using a tunnel or by cupping it on the open hand, it will experience less anxiety and stress when compared to a mouse that is gripped and lifted at the base of its tail (Hurst and West, 2010). The benefits for both animal welfare and quali- ty of research data associated with tunnel and cup handling appear to have recently convinced many stakeholders: More and more working groups have adopted tunnel and cup handling in their laboratory routines, and even entire institutions are considering to replace tail handling with the refined mouse handling techniques.

To introduce non-aversive mouse handling at the Research Facilities for Experimental Medicine (FEM), Charité - Universitätsmedizin Berlin, three courses on non-aversive mouse handling techniques were offered in November and December 2019. Each course had the same content and comprised a 40-min lecture on tunnel and cup handling in mice and approximately 20 min discussion. All staff having contact with laboratory mice, i.e., animal caretakers, technical assistants, scientists, and veterinarians, had the opportunity to attend one of the courses and
Received May 12, 2020;

(c) The Authors, 2020.

ALTEX 37(4), 671-674. doi:10.14573/altex.2005121

Correspondence: Katharina Hohlbaum, PhD

Freie Universität Berlin, Department of Veterinary Medicine

Institute of Animal Welfare, Animal Behavior, and Laboratory Animal Science

Königsweg 67 (Haus 21)

14163 Berlin, Germany

(katharina.hohlbaum@fu-berlin.de)
This is an Open Access article distributed under the terms of the Creative Commons Attribution 4.0 International license (http://creativecommons.org/licenses/by/4.0/) which permits unrestricted use, distribution and reproduction in any medium, provided the original work is appropriately cited. 
thoroughly discuss the non-aversive mouse handling techniques with their colleagues, the animal welfare officers, and the animal facility manager.

The participants of the course were introduced to tunnel and cup techniques using videos produced by Jane Hurst and the NC3Rs ${ }^{1}$. Mice can be familiarized with tunnel handling during cage cleaning, whereas it is a little more effort to habituate them to cup handling (Gouveia and Hurst, 2019). A broad overview on the effects of non-aversive handling techniques on a range of parameters was presented to the course participants: They learned that tunnel and cup handling reduced aversion towards the handling device in different mouse strains, i.e., BALB/c, C57BL/6 and ICR(CD-1), when compared to tail handling (Hurst and West, 2010). It is assumed that this was independent of the person handling the mice, as shown for female BALB/c mice (Hurst and West, 2010). Interestingly, the positive effects are not reversed by procedures, e.g., abdominal inspections, scruff handling, subcutaneous injections, tattooing or ear tagging (Gouveia and Hurst, 2019; Hurst and West, 2010; Roughan and Sevenoaks, 2019) that are carried out after the mouse has been picked up in a tunnel. Mice urinate and defecate less during the handling session when tunnel or cup techniques are applied, indicating lower stress levels in comparison to mice handled by tail (Hurst and West, 2010; Nakamura and Suzuki, 2018). Moreover, a number of studies have confirmed that the refined handling techniques reduce anxiety-related behavior using the elevated plus maze and the open field test (Ghosal et al., 2015; Hurst and West, 2010; Nakamura and Suzuki, 2018; Clarkson et al., 2018). Tail-handled mice show a more depressive-like state with regard to anhedonic responses than tunnel-handled mice (Clarkson et al., 2018). Since anxiety and stress influence the performance of animals in behavioral tests, tunnel and cup handling therefore contribute to more robust research data. Gouveia and Hurst demonstrated that the refined handling techniques improved the performance of mice in comparison to tail handling in the habituation-dishabituation paradigm (Gouveia and Hurst, 2017), i.e., handling techniques have a significant impact on the reliability of performance in behavioral tests. Besides behavioral parameters, handling methods can influence blood corticosterone and glucose levels (Ghosal et al., 2015; Ono et al., 2016).

After the course, participants could voluntarily fill out a paper-based questionnaire on their opinion on the non-aversive mouse handling techniques. The mixed type questionnaire contained six items with a 6-point Likert type scale measuring agreement, two open-ended questions and, finally, demographics. The survey aimed to provide an insight into the acceptance and concerns of the animal facility and scientific staff in order to consider all perspectives and identify potential problems that may be associated with the implementation of this refinement strategy.

As answering the questions in the survey was optional, not all participants filled in all questions. Therefore, the number of persons who gave an answer to a question is given in brackets below. Out of 267 registered participants 113 participants took part in the survey. Participants were on average 35 years old ( \pm 9 standard deviation; 72 persons) and had approximately 9 years experiences ( \pm 7 standard deviation; 86 persons) in the field of animal experimentation. Most of the participants stated that they work in animal experimental units $(n=89)$. Only a small number indicated that they work mainly in animal husbandry and breeding units (20 persons).

Figure 1 shows the participants' opinion on tunnel and cup handling after they attended the course on non-aversive mouse handling techniques. The participants were asked to what extent they agreed with the statements A (103 responses), B (104 responses), C (106 responses), and D (107 responses). Almost everyone agreed (fully to somewhat) that tunnel and cup handling improved the well-being of mice (99\%) and the human-animal relationship (96\%) (Fig. 1A,B). Most of the participants indicated that the initial additional expenditure of time is justifiable (78\%) (Fig. 1C). Assuming that both humans and animals are familiar with tunnel and cup handling, 64\% of the participants found that the amount of work required did not differ between the refined handling and tail handling techniques. However, 16\% somewhat, $16 \%$ generally, and $4 \%$ fully disagreed with this statement.

Statements referring to the respective fields of activity, i.e., animal husbandry and breeding unit as well as experimentation unit, were rated differently by staff from different units (Fig. 2). The participants were asked to what extent they agreed with the statements E (19 responses from employees at animal husbandry and breeding units, 79 responses from employees at animal experimental units) and $\mathrm{F}$ (16 responses from employees at animal husbandry and breeding units, 77 responses from employees at animal experimental units). Most of the respondents, i.e., $58 \%$ persons from the animal husbandry and breeding units and $86 \%$ from animal experimental units, believe that it is possible to implement tunnel and cup handling in their work area. $78 \%$ of the staff at the experimental units but only $50 \%$ of the staff at animal husbandry and breeding units expected scientific results to improve if tunnel and cup handling were implemented in their units.

The survey also offered the participants the opportunity to indicate problems they anticipate when implementing tunnel and cup handling. The answers to this open-ended question were categorized and then assigned to the following five statements (the number in brackets is the number of participants giving the respective response):

1) Non-aversive handling techniques increase expenditure of time and costs $(n=15)$.

2) Non-aversive handling techniques increase bites and escapes $(\mathrm{n}=10)$.

3) Non-aversive handling techniques should be used mainly by animal caretakers $(n=3)$.

4) Non-aversive handling techniques should be used by everyone $(n=8)$.

5) Non-aversive handling techniques make health control more difficult $(n=6)$.

1 www.nc3rs.org.uk/video-clips 


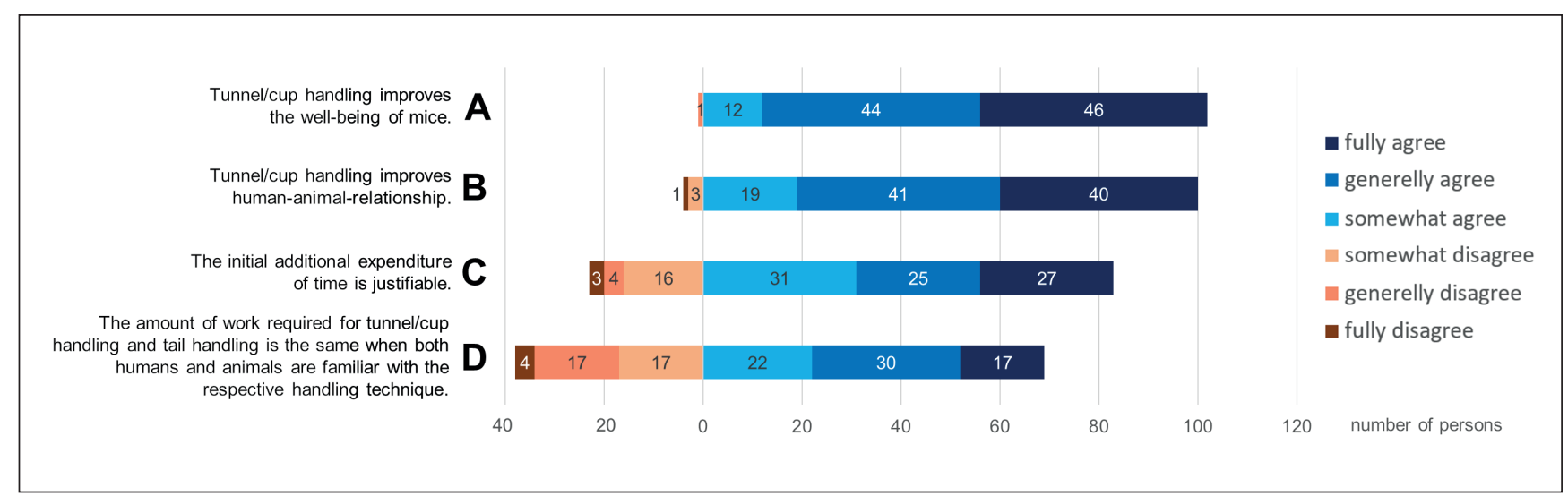

Fig. 1: Participants' opinion on non-aversive mouse handling

A was answered by 103 , B by 104, C by 106, and D by 107 participants. Since both lecture and survey were in German, results of the survey were translated into English for this article.

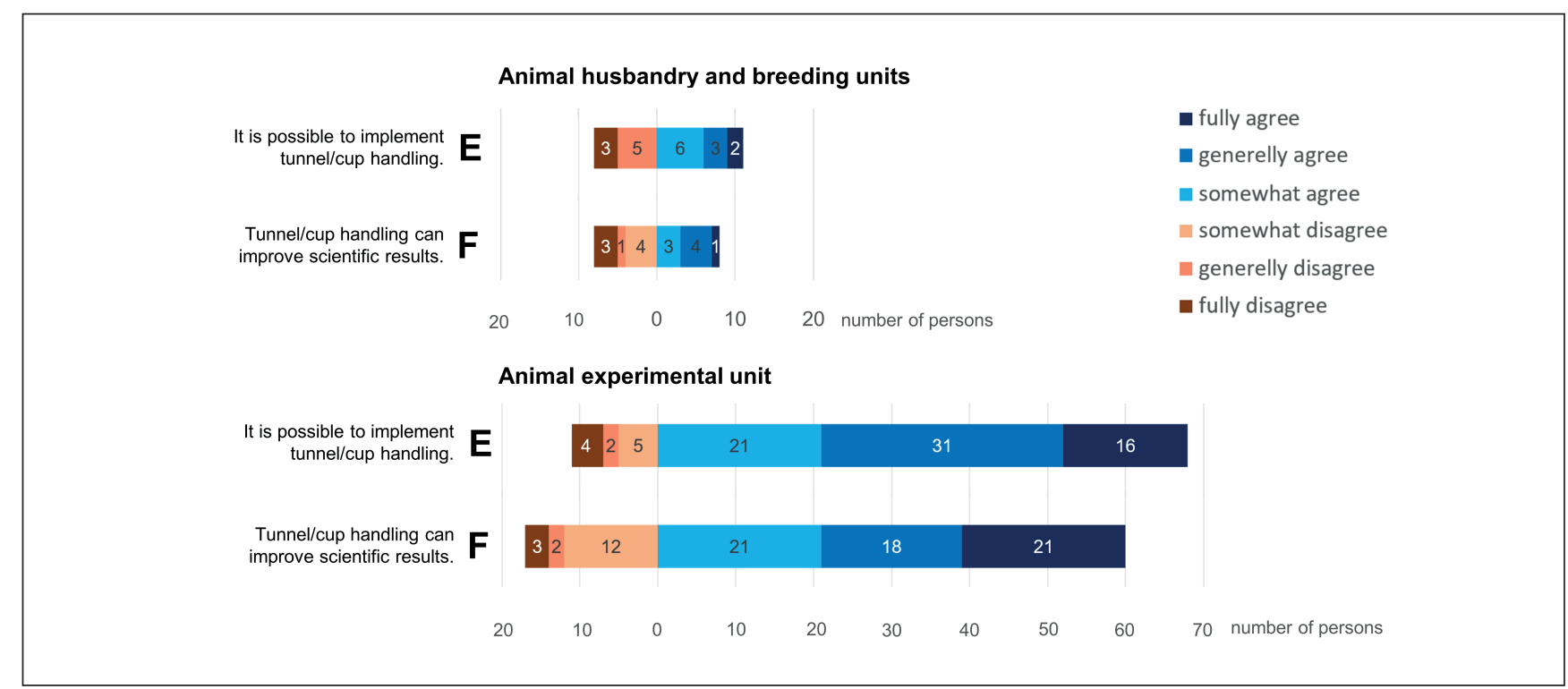

Fig. 2: Participants' opinion on the implementation of non-aversive mouse handling and its impact on scientific quality $E$ and $F$ were answered by 19 and 16 persons from animal husbandry and breeding units as well as by 79 and 77 persons from animal experimental units, respectively. Since both lecture and survey were in German, results of the survey were translated into English for this article.

The time factor appeared to be the main problem: 15 participants feared that the refined handling techniques would increase expenditure of time and costs. Of course, there are additional costs for the tunnels, which can be either purchased from commercial suppliers or made from acrylic glass tubes. Since tunnels, however, also serve as enrichment, i.e., as shelters, when left in the cages, animal facilities should have a certain budget for this kind of acquisition. The concern that cage cleaning may take considerably longer when using non-aversive handling techniques is addressed by Gouveia and Hurst (2019). They demonstrated that mice could be familiarized with the tunnel when they were handled using the tunnel for about 2 seconds during fortnightly cage cleaning only. After the first cage change, tunnel handling significantly in- creased the voluntary interaction with the experimenter's hand and decreased the reluctance to be handled when compared to tail handling (Gouveia and Hurst, 2019). For cup handling, similar effects were only found when the animals were handled daily for an additional 2 seconds between the fourth and fifth cage clean (Gouveia and Hurst, 2019). Against this background, habituation of mice to tunnel handling by persons familiar with this method requires only little if any additional expenditure of time. However, in a transgenic mouse breeding colony with different strains, ages, sexes, and cage densities, Doerning et al. (2019) found that animal caretakers who were not familiar with the non-aversive handling methods but with forceps changed cages faster when using forceps or cup handling than when using tunnels. They did 
not find a decrease in cage-change speed over the four fortnightly cage change sessions, suggesting that the acclimation effect was delayed and set in after the study period. To train the animal facility and scientific staff in using the non-aversive handling techniques may take a few days or weeks - probably similar to the time it takes to learn how to pick up a mouse by its tail or using forceps. If tunnel and cup handling were taught in the training of animal caretakers and laboratory animal science courses and used from the beginning of the professional career, the habituation phase of the staff would be omitted. In this scenario, everyone would have learnt the refined handling techniques during basic training and, therefore, the question whether everyone or only animal caretakers should apply tunnel or cup handling would no longer arise. We should also remember at this point that animal welfare must not be compromised to save labor, time, or costs according the German Animal Welfare Act.

Although a transparent tunnel allows observation of the mice within the tunnel, some participants claimed that the use of tunnels would make health control of mice more difficult. However, it was shown that the ability of the staff to detect abnormalities at cage change was not inhibited by tunnel or cup handling; moreover, tunnels left in the home cages did not impair cage-side health observations (Doerning et al., 2019).

Ten participants expressed the fear that the mice would attempt to bite or escape. Whether this is true has not been extensively investigated yet. From the authors' view, the reverse should apply, since animals will rather attempt to bite or escape if they experience anxiety or stress in response to human interaction. However, non-aversive handling techniques decrease anxiety-related behavior (Ghosal et al., 2015; Hurst and West, 2010; Nakamura and Suzuki, 2018; Clarkson et al., 2018).

Moreover, some participants wondered whether non-aversive handling works on all mouse strains and models. Models for infection were given as an example of exceptions where the refined handling may not be applied. The participants were also interested in effects of tunnel and cup handling in large-scale or long-term studies. Doerning et al. (2019) also requested further investigations on labor efficiency. Based on these questions, the authors of the present work decided to collect data on the practicability in breeding and husbandry units with a variety of different strains.

All in all, the course on non-aversive handling techniques provided useful insight into the acceptance and concerns of the animal facility and scientific staff. Since the successful implementation of a new refinement method depends on everyone pulling in the same direction, this strategy may serve as a first step towards a stepwise implementation of tunnel and cup handling as part of a culture of care.

\section{References}

Bailey, J. (2017). Does the stress inherent to laboratory life and experimentation on animals adversely affect research data? Altern Lab Anim 45, 299-301. doi:10.1177/026119291704500605

Balcombe, J. P., Barnard, N. D. and Sandusky, C. (2004). Laboratory routines cause animal stress. Contemp Top Lab Anim Sci 43, 42-51. https://bit.ly/2ZX2LbJ (last accessed on 12 May 2020)

Clarkson, J. M., Dwyer, D. M., Flecknell, P. A. et al. (2018). Handling method alters the hedonic value of reward in laboratory mice. Sci Rep 8, 2448. doi:10.1038/s41598-018-20716-3

Doerning, C. M., Thurston, S. E., Villano, J. S. et al. (2019). Assessment of mouse handling techniques during cage changing. J Am Assoc Lab Anim Sci 58, 767-773. doi:10.30802/aalasjaalas-19-000015

Ghosal, S., Nunley, A., Mahbod, P. et al. (2015). Mouse handling limits the impact of stress on metabolic endpoints. Physiol Behav 150, 31-37. doi:10.1016/j.physbeh.2015.06.021

Gouveia, K. and Hurst, J. L. (2017). Optimising reliability of mouse performance in behavioural testing: The major role of non-aversive handling. Sci Rep 7, 44999. doi:10.1038/ srep44999

Gouveia, K. and Hurst, J. L. (2019). Improving the practicality of using non-aversive handling methods to reduce background stress and anxiety in laboratory mice. Sci Rep 9, 20305. doi:10.1038/s41598-019-56860-7

Hurst, J. L. and West, R. S. (2010). Taming anxiety in laboratory mice. Nat Methods 7, 825-826. doi:10.1038/nmeth. 1500

Nakamura, Y. and Suzuki, K. (2018). Tunnel use facilitates handling of ICR mice and decreases experimental variation. $J$ Vet Med Sci 80, 886-892. doi:10.1292/jvms.18-0044

Ono, M., Sasaki, H., Nagasaki, K. et al. (2016). Does the routine handling affect the phenotype of disease model mice? Jpn J Vet Res 64, 265-271. https://bit.ly/2EgoL94 (last accessed on 12 May 2020)

Roughan, J. V. and Sevenoaks, T. (2019). Welfare and scientific considerations of tattooing and ear tagging for mouse identification. J Am Assoc Lab Anim Sci 58, 142-153. doi:10.30802/ aalas-jaalas-18-000057

\section{Acknowledgements}

We thank Ramona Warnke for assistance in the course on non-aversive mouse handling techniques. This work was financially supported by the Charité 3R, Charité - Universitätsmedizin Berlin. The funder had no role in study design, data collection and analysis, decision to publish, or preparation of the manuscript. 